\title{
Reproducibility and Clinical Correlates of Supine Diaphragmatic Motion Measured by M-Mode Ultrasonography in Healthy Volunteers
}

\author{
Simone Scarlata Damiana Mancini Alice Laudisio Augusto Benigni \\ Raffaele Antonelli Incalzi \\ Unit of Respiratory Pathophysiology and Thoracic Endoscopy, Division of Geriatrics, Campus Bio Medico University \\ and Teaching Hospital, Rome, Italy
}

\section{Keywords}

Diaphragmatic motion M-mode ultrasonography · Intra-observer agreement · Inter-observer agreement .

Quiet breathing·Deep breathing

\begin{abstract}
Background: Assessing diaphragm mobility is important to detect malfunctions or impending exhaustion and to evaluate the effects of many chest and abdominal conditions on respiratory mechanics. Amongst several imaging methods, ultrasonography represents the only noninvasive, nonionizing imaging technique widely available for the direct assessment of diaphragm excursion. Objectives: the aim of this study is to prospectively assess the supine diaphragmatic motion amplitude, intra- and inter-observer agreement, and anthropometric correlates of diaphragm motion variability, measured through M-mode ultrasonography, in a sample of healthy volunteers. Methods: Onehundred healthy volunteers were considered eligible for the study. Instead of B-mode imaging, the M-mode technique was used to achieve a more accurate measurement
\end{abstract}

\section{KARGER}

(c) 2018 S. Karger AG, Basel

E-Mail karger@karger.com

www.karger.com/res of diaphragm motion. To assess intra-observer variability, 3 consecutive measurements ( $M$-mode and B-mode) of the right dome motion were obtained at every session. To test for inter-rater reliability, the subjects were asked to provide 2 more diaphragm motion measurements every week, each performed by 2 experienced operators, and 42 subjects accepted. Results: Diaphragmatic motion was positively correlated with height and weight both at quiet (Spearman's coefficient $=0.514, p<0.001$ and $0.314, p=$ 0.038 ) and deep breathing (Spearman's coefficient $=0.342$, $p=0.001$ and $0.225, p=0.024$, respectively) and negatively correlated with age, but only during deep breathing (Spearman's coefficient $=-0.272, p=0.006$ ). Intra-observer agreement degree on all 3 measurements was excellent during both quiet and deep breathing, with a Cronbach's alpha of 0.793 and 0.901 , respectively, and an intra-class coefficient of 0.797 and 0.900 , respectively. Similarly, the degree of inter-observer agreement achieved a Cronbach's alpha of 0.638 and 0.776 , and an inter-class coefficient of 0.632 and 0.778 , respectively. Deep breathing was associated with sex only in linear multivariable models $(B=-10.14 ; 95 \%$ confidence interval $[\mathrm{Cl}]-17.86,-2.41 ; p=0.011)$, while qui- 
et breathing resulted to be affected by height only ( $\mathrm{B}=$ 30.05; 95\% Cl 0.79-59.31; $p=0.044)$. Conclusions: Diaphragm excursion measurements using the M-mode technique were accurate and could be reproduced also when obtained in recumbent patients. After adjustments, the main predictors of diaphragmatic motion were sex and height, which should be considered to design a specifically tailored study and to develop normality reference equations.

(c) 2018 S. Karger AG, Basel

\section{Introduction}

The diaphragm is the main muscle involved in breathing. Assessing its mobility is important to detect malfunctions or impending exhaustion and to evaluate the effects of many chest and abdominal conditions on respiratory mechanics [1]. Diaphragm dysfunction is associated with respiratory insufficiency and poorer outcomes [2].

Several imaging methods were suggested to carry out diaphragm mobility assessment: fluoroscopy [3], computed tomography [4], magnetic resonance imaging [5], and chest radiography [6]. Each technique presents specific strengths and limitations, mainly relating to cost, radiation exposure, required expertise, and method availability [7].

Ultrasonography instead represents the only noninvasive, nonionizing imaging technique widely available to directly assess diaphragmatic functioning [8]. A specific sonographic approach allows to assess muscle mobility through excursion measurement of the diaphragm dome. Thanks to ultrasound versatility, diaphragm morphology and function can be assessed in various settings, such as the outpatient clinic, patient's bedside, hospital departments, and intensive care units. Though different approaches were proposed, a comprehensive sonographic examination was never standardized for clinical use, nor was the reliability of this approach tested in every setting. We are aware of only a few previous studies providing intra- and inter-rater reproducibility and normal values in standing and semirecumbent patients $[9,10]$, but we could not find studies assessing the validity and reliability of diaphragmatic ultrasound in decumbent patients.

The aim of this study is to prospectively assess supine diaphragm motion amplitude, intra- and inter-observer agreement, and anthropometric correlates of diaphragm motion variability, measured through M-mode ultrasonography, in a sample of healthy volunteers.
Table 1. Demographic, anthropometric, and lifestyle features of the study population $(N=100)$
Males, $n(\%)$

Mean age (SD), years

Mean weight (SD), $\mathrm{kg}$

Mean height (SD), $\mathrm{m}$

Mean BMI (SD)

Smoking exposure, $n(\%)$

Sedentary work, $n(\%)$

Physically active, $n$ (\%)
$49 / 100(49)$

$40(15)$

$71.2(14.6)$

$1.70(0.1)$

$24.4(3.8)$

$21 / 100(21)$

$56 / 100(56)$

$47 / 100(47)$

\section{Materials and Methods}

One-hundred and 8 consecutive healthy volunteers were enrolled in the study. Out of these, 100 were considered eligible. When the local ethic committee approved the study protocol (Prot. 30/17. PAR ComEt CBM), all subjects provided a signed written informed consent form. The trial eligibility criteria included asymptomatic healthy adults; absence of chronic pain or acute symptomatology during the $72 \mathrm{~h}$ preceding the session; and no diagnosed pathology. Exclusion criteria were pregnancy; breastfeeding; diagnosis of pathological conditions; chronic drug treatments; and a medical history of abdominal surgery.

After enrollment in the study, a comprehensive clinical history was obtained and a physical examination was performed, in order to identify any active or relevant clinical conditions. When available, blood analysis and medical records were also reviewed.

\section{Diaphragm Ultrasound Assessment}

All participants received 3 ultrasound evaluations (carried out using the ultrasound imaging system Exagyne, produced by ECM [Echo Control Medical] in Angoulême, France) on diaphragm motion at a 7 -day time lapse. Using a convex probe and frequencies between 2.5 and $3.5 \mathrm{mHz}$, subcostal scans were taken during subjects' quiet $(\mathrm{QB})$ and deep $(\mathrm{DB})$ breathing (inspiratory reserve capacity), starting from functional residual capacity and according to described protocols [8]. The transducer was placed right and anterior to the midclavicular line; the gallbladder and the inferior cave vein were considered as the anatomical landmarks for obtaining a conventional transverse section in B-mode scans [10]. The $\mathrm{M}$-mode technique pattern for diaphragm motion was used to achieve a more accurate measurement compared to B-mode images.

To assess intra-observer variability, volunteers were examined twice by the same operator. At every session, 3 consecutive Mmode and $\mathrm{B}$-mode right dome motion measurements were taken systematically, both at $\mathrm{QB}$ and $\mathrm{DB}$. In order to test for inter-rater reliability, the subjects were asked to provide 2 more diaphragm motion measurements every week, and 42 subjects accepted. In turn, 2 experienced operators carried out the ultrasound re-evaluation on every participant.

\section{Statistical Analysis}

Statistical analyses were performed using SPSS software (version 23.0; produced by SPSS Inc., Chicago, IL, USA). All data are expressed as means \pm standard deviations (SD) or $95 \%$ confi- 
Table 2. Right diaphragmatic excursion in males and females

\begin{tabular}{lllllllr}
\hline & Overall & $95 \%$ CI & Males & $95 \%$ CI & Females & $95 \%$ CI & $p$ value \\
\hline QB, mm & $17.6(5.4)$ & $16.0-19.2$ & $20.3(5.7)$ & $17.7-22.9$ & $15.1(3.7)$ & $13.5-16.7$ & 0.001 \\
DB, mm & $62.0(15.5)$ & $57.3-66.7$ & $69.3(14.6)$ & $62.6-75.9$ & $55.4(13.3)$ & $49.6-61.2$ & $<0.001$ \\
\hline
\end{tabular}

Values are presented as mean (SD).

dence intervals (CI), as median and interquartile range for continuous variables, or as percentages for categorical variables. The data distribution was analyzed using a Kolmogorov-Smirnov test. Differences between males and females were assessed through Student's $t$ test or the Mann-Whitney test, as appropriate. Spearman's correlation analysis was adopted to establish the correlation between the variables. Differences were considered significant at $p<0.05$.

The association of ultrasonographic measurements with demographic and anthropometric data was assessed using a linear regression analysis, while variables associated in univariate analyses were entered in multivariable models. Abnormally distributed variables were analyzed after log transformation. Intra-class correlation coefficient (ICC) and Bland-Altman plotting were calculated for the assessment of intra- and inter-rate reproducibility [11].

\section{Results}

The data obtained from 100 subjects were finally analyzed. The mean age of the participants was $40.1 \pm 14.9$ years; 49 were males; 21 out of 100 had a current or former cigarette-smoking exposure, while 56 and 53, respectively, reported a sedentary work profile and/or to be physically inactive. Table 1 shows further demographic, anthropometric, and lifestyle characteristics of the study population.

The correct right dome ultrasound visualization was achieved in all participants. Overall, the mean diaphragm excursion measurements were $17.6 \pm 5.4 \mathrm{~mm}$ (95\% CI 16.0-19.2) at QB and $62.0 \pm 15.5 \mathrm{~mm}$ (95\% CI 57.3-66.7) at DB. Diaphragm motion varied significantly on a gender basis, with males presenting $20.3 \pm 5.7$ versus $15.1 \pm 3.7 \mathrm{~mm}$ in females $(p=0.001)$ at $\mathrm{QB}$, and $66.7 \pm 14.0$ versus $55.3 \pm 12.6 \mathrm{~mm}$ at $\mathrm{DB}(p<0.001)$, respectively (Table 2 ).

Diaphragm motion correlated positively with height and weight both at QB (Spearman's correlation coefficient $=0.514, p<0.001$ and $0.314, p=0.038)$ and at $\mathrm{DB}$ (Spearman's correlation coefficient $=0.342, p=0.001$ and $0.225, p=0.024$, respectively), and negatively with age,

Reproducibility and Clinical Correlates of Supine Diaphragmatic Motion
Table 3. Correlation analysis between diaphragmatic excursion and anthropometric data

\begin{tabular}{llrr}
\hline & & $\begin{array}{l}\text { Spearman's correlation } \\
\text { coefficient (R) }\end{array}$ & $p$ value \\
\hline QB & Age & -0.227 & 0.139 \\
& Height & 0.514 & $<0.001$ \\
& Weight & 0.314 & 0.038 \\
DB & Age & -0.272 & 0.006 \\
& Height & 0.342 & 0.001 \\
& Weight & 0.225 & 0.024 \\
\hline
\end{tabular}

but only during DB (Spearman's correlation coefficient = $-0.272, p=0.006$ ) (Table 3 ). The scatter plots representing these data can be found in Figure 1.

The intra-observer agreement degree on all 3 measurements was excellent during both $\mathrm{QB}$ and $\mathrm{DB}$, with a Cronbach's alpha of 0.793 and 0.901 respectively, and an intraclass coefficient of 0.797 and 0.900 , respectively. Similarly, the degree of inter-observer agreement achieved a Cronbach's alpha of 0.638 and 0.776 , with an inter-class coefficient of 0.632 and 0.778 , respectively. These results, together with all correlation matrices, are listed in Table 4. Figures 2 and 3 show the Bland-Altman plots highlighting the differences between the measurements and their mean values at $\mathrm{QB}$ and $\mathrm{DB}$.

$\mathrm{DB}$ was associated with sex only in linear multivariable models $(\mathrm{B}=-10.14 ; 95 \% \mathrm{CI}-17.86$ to $-2.41 ; p=0.011$; Table 5), while QB resulted to be affected by height only ( $\mathrm{B}=30.05 ; 95 \%$ CI 0.79-59.31; $p=0.044$; Table 5).

\section{Discussion}

This study provides evidence that right dome excursion measurements using the M-mode technique can be reproduced also in recumbent patients. The high coef- 


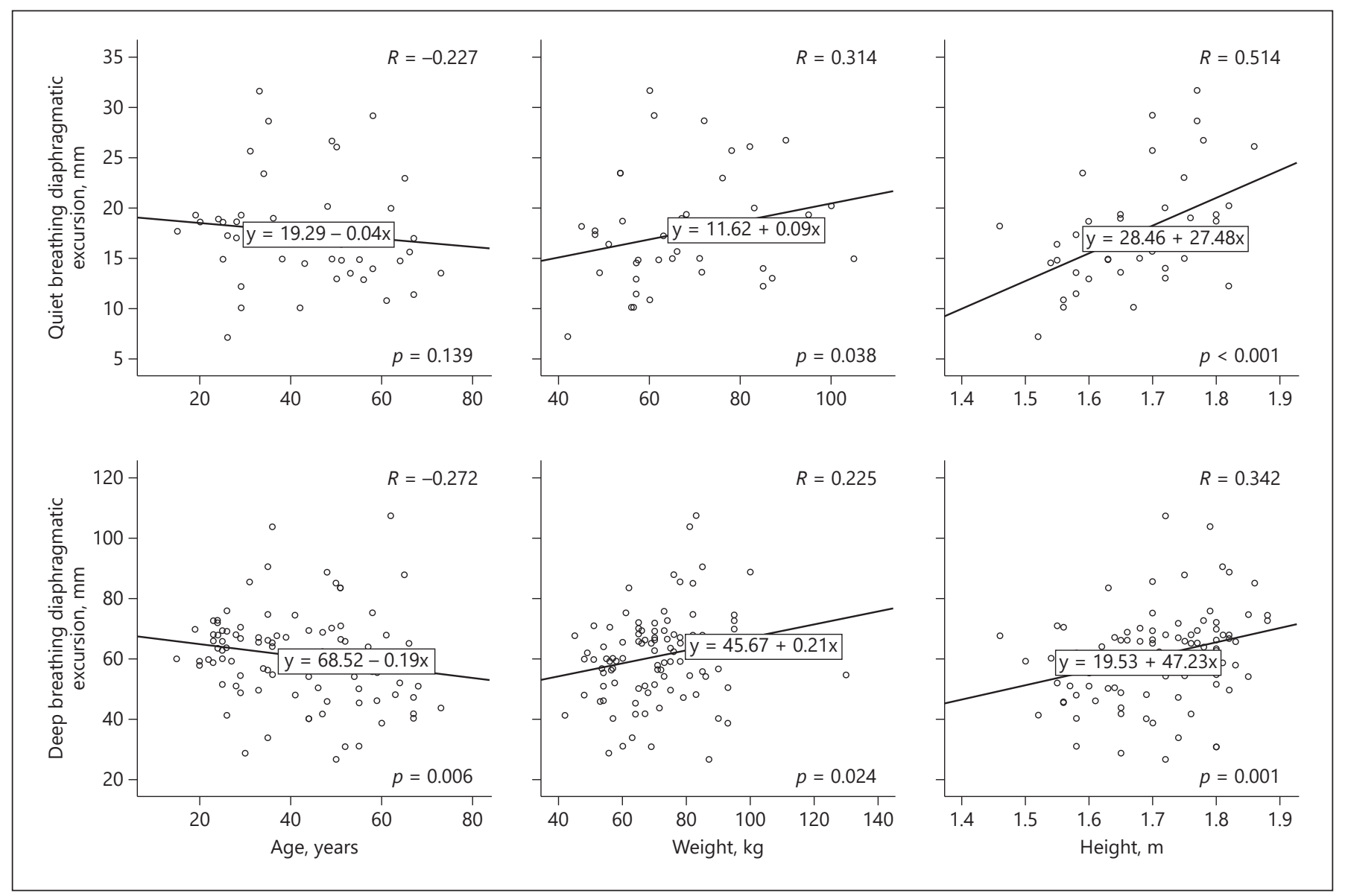

Fig. 1. Scatter plots representing the correlation of height, weight, and age with diaphragmatic motion at quiet and deep breathing, respectively.

Table 4. ICCs in the study population

\begin{tabular}{|c|c|c|c|c|c|}
\hline & \multicolumn{2}{|c|}{ Cronbach's alpha } & \multicolumn{2}{|l|}{ Correlation matrix } & ICC \\
\hline \multicolumn{3}{|c|}{ Intra-rater agreement } & II ${ }^{\circ}$ measurement & $I I I^{\circ}$ measurement & \\
\hline \multirow[t]{3}{*}{ QB } & 0.793 & $\mathrm{I}^{\circ}$ measurement & 0.528 & 0.409 & 0.797 \\
\hline & & $\mathrm{II}^{\circ}$ measurement & 1.000 & 0.738 & \\
\hline & & & $I I^{\circ}$ measurement & III ${ }^{\circ}$ measurement & \\
\hline \multirow[t]{2}{*}{$\mathrm{DB}$} & 0.901 & $\mathrm{I}^{\circ}$ measurement & 0.743 & 0.711 & 0.900 \\
\hline & & $\mathrm{II}^{\circ}$ measurement & 1.000 & 0.807 & \\
\hline \multicolumn{3}{|c|}{ Inter-rater agreement } & $I I^{\circ}$ measurement & III $I^{\circ}$ measurement & \\
\hline \multirow[t]{3}{*}{ QB } & 0.638 & $\mathrm{I}^{\circ}$ measurement & 0.303 & 0.235 & 0.632 \\
\hline & & $\mathrm{II}^{\circ}$ measurement & 1.000 & 0.580 & \\
\hline & & & $I I^{\circ}$ measurement & $I I I^{\circ}$ measurement & \\
\hline \multirow[t]{2}{*}{$\mathrm{DB}$} & 0.776 & $\mathrm{I}^{\circ}$ measurement & 0.652 & 0.362 & 0.778 \\
\hline & & $\mathrm{II}^{\circ}$ measurement & 1.000 & 0.608 & \\
\hline
\end{tabular}




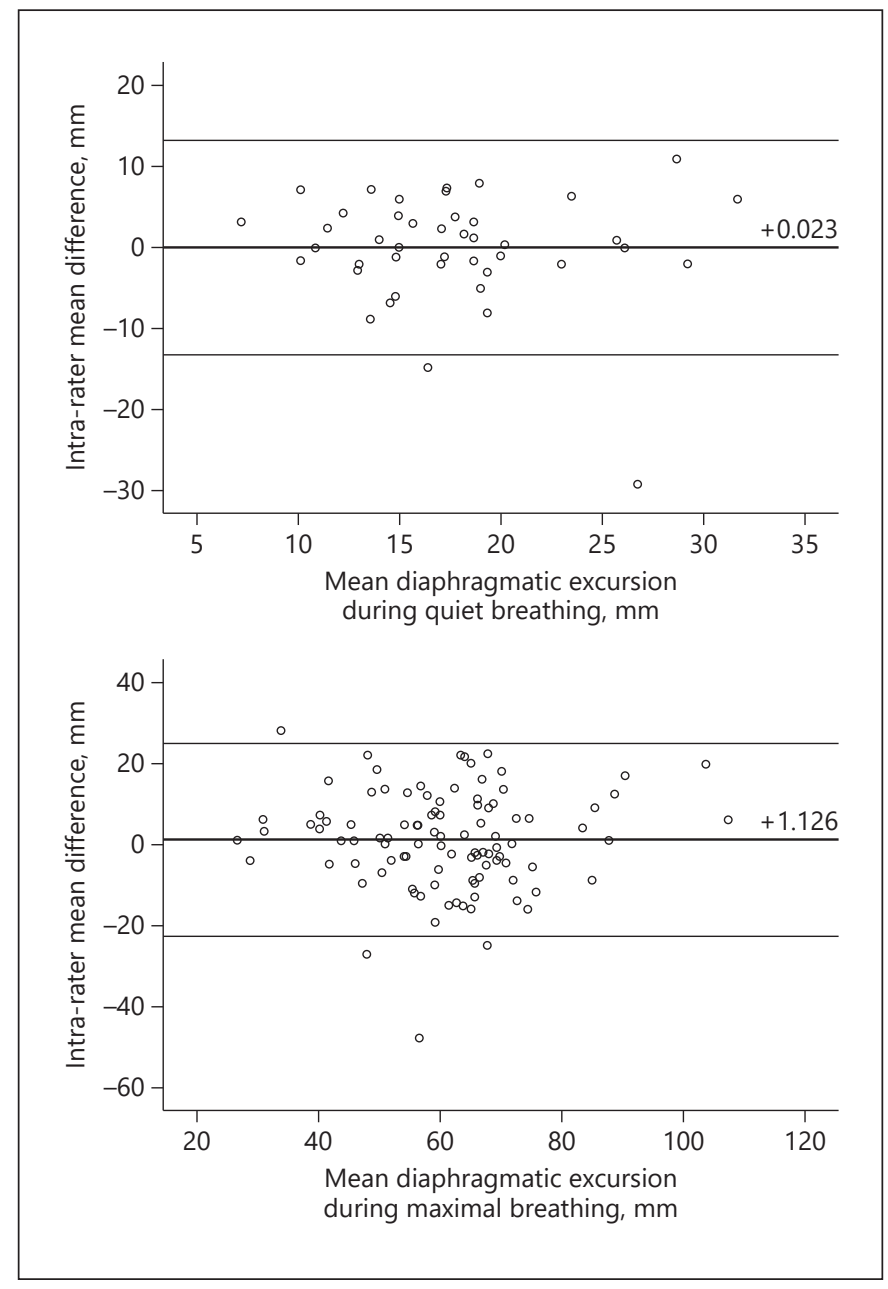

Fig. 2. Bland-Altman plots showing intra-rater agreement at quiet and maximal breathing, respectively.

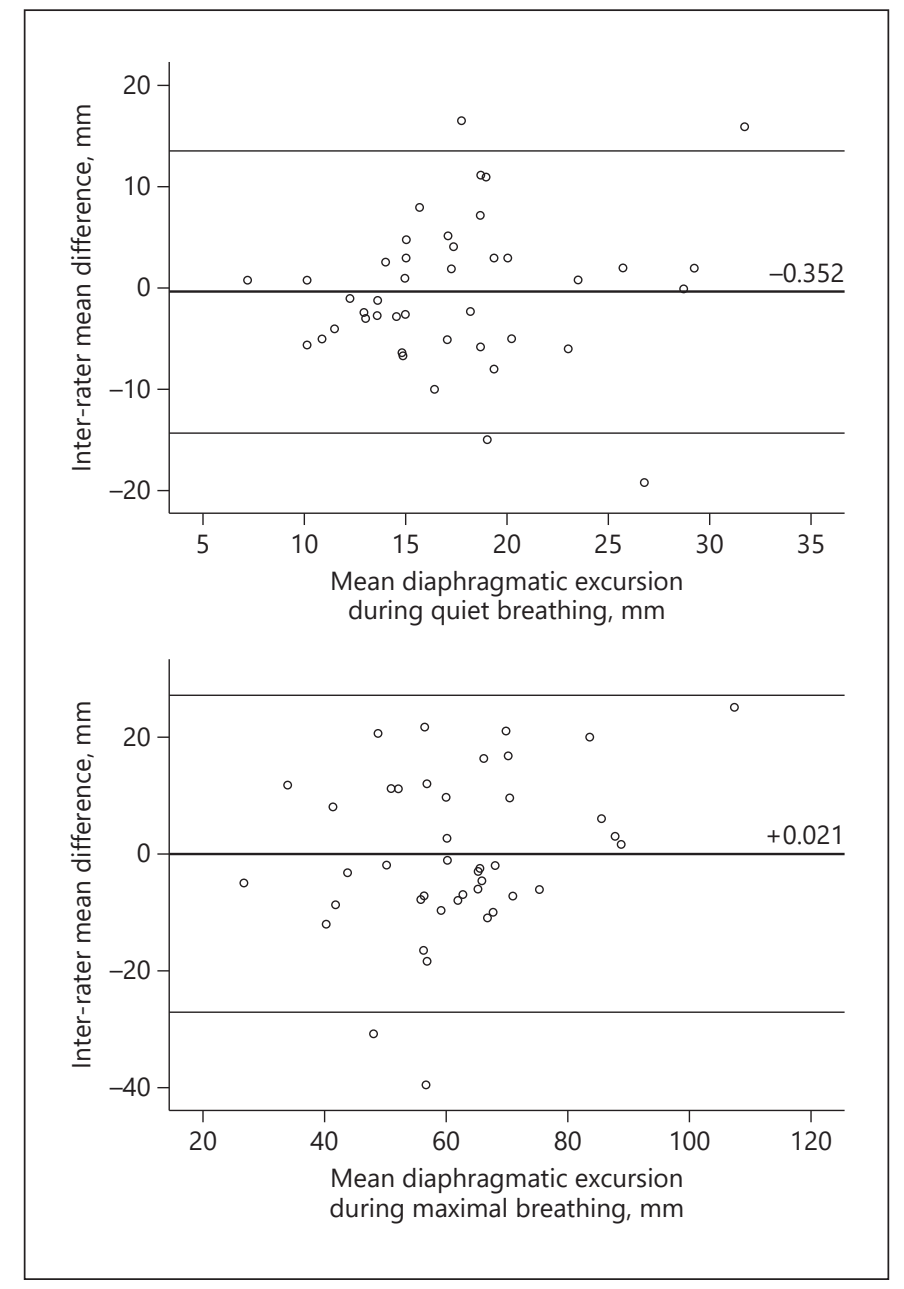

Fig. 3. Bland-Altman plots showing inter-rater agreement at quiet and maximal breathing, respectively.

Table 5. Linear univariate and multivariable models of demographic and anthropometric determinants of diaphragm excursion at DB and QB

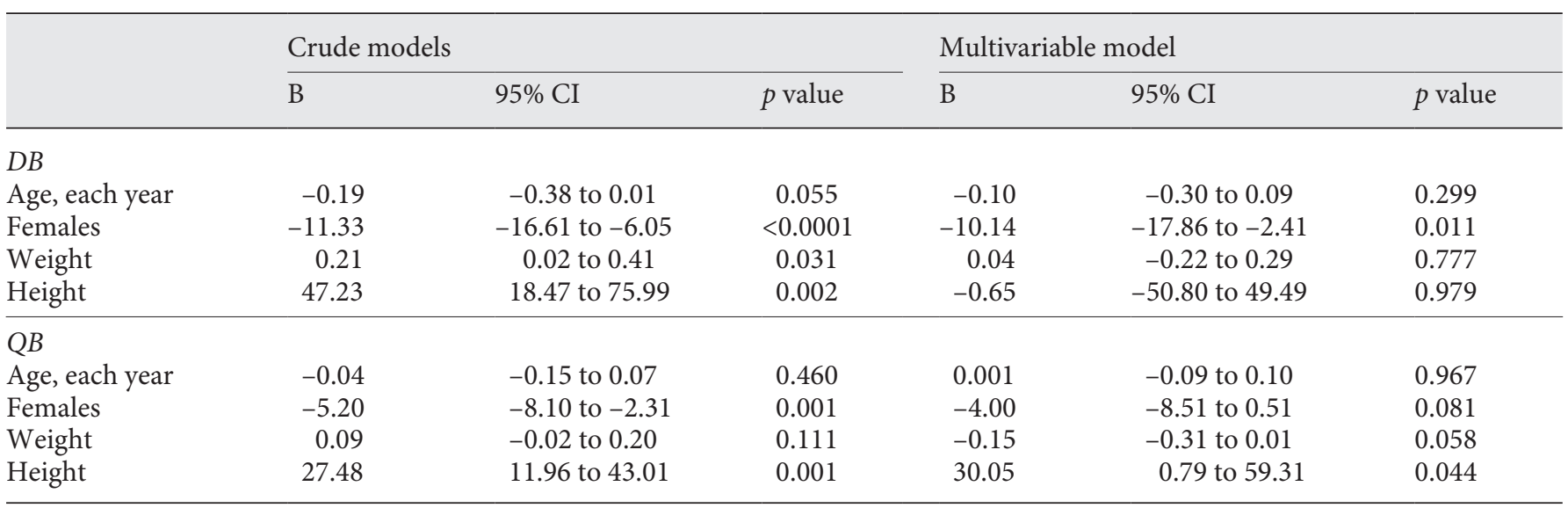


ficient correlation and the low mean differences observed in the Bland-Altman plots, both within and between observers, as well as the robust Cronbach's alpha and inter- and intra-class coefficients we have found (Table 4) support this conclusion, thus confirming the potential of using ultrasound technology to assess diaphragmatic function in clinical practice. Indeed, although both inter- and intra-rater diaphragm measures resulted to be highly reproducible (overall Cronbach's alpha and ICC $>0.6$ ), inter-rater correlation coefficients showed, in a few one-on-one comparisons, values $<0.6$. This was particularly true during QB, indicating that, due to the wide variability of the breathing pattern at rest, a standardized breathing maneuver should be developed to minimize the risk of error.

Normal limits were established from these measurements that were comparable with those reported in other studies using both ultrasonography $[8,10]$ and alternative methods (such as fluoroscopy [3]), although those were obtained in standing or semirecumbent subjects. We analyzed a representative sample of the healthy population with a normal distribution of the main anthropometric parameters. This allowed us to investigate whether these indexes could be associated with diaphragmatic excursion and create prediction models. It is not the first time that a precision study is carried out with this purpose. Available data, however, result inconclusive, since technical issues were raised [12] and conflicting data currently exist [10]. We found the fifth percentile of diaphragm motion to be 17.7 and $13.5 \mathrm{~mm}$ at $\mathrm{QB}$, and 62.6 and $49.6 \mathrm{~mm}$ at $\mathrm{DB}$ for the male and female population, respectively. The lower limits might be used as cutoff points for diagnosing diaphragmatic weakness or overt paralysis. Further research would be needed to test whether there is a correspondence between diaphragm motion below the 5th percentile and clinical criteria for weakness and/or paralysis.

As expected, gender influences diaphragm motion, showing larger excursions in males than in females; it is then likely that part of this variability is due to sex-related differences in anthropometric indexes, since height and weight are linearly correlated with diaphragm motion and differ significantly in the 2 groups. Several other mechanisms, such as differences in the muscle mass/ force ratio, perfusion, contractile properties, and metabolism [13] should, however, be taken into account when analyzing correlations. Age displays a weak inverse linear correlation only during $\mathrm{DB}$, while no significant association was found at QB. This is in accordance with the findings of Verschakelen and Demedts [14], who have determined thoracoabdominal movements by inductive pletismography in several body postures, including the supine one.

We found a mean diaphragm excursion of $17.6 \pm 5.4$ $\mathrm{mm}(95 \% \mathrm{CI} 16.0-19.2)$ at QB and of $62.0 \pm 15.5 \mathrm{~mm}$ (95\% CI 57.3-66.7) during DB. These values are in line with what was assessed in other studies $[3,15]$. Moreover, previous studies suggested a relationship between the inhaled volume and body position, supposing hemidiaphragm movements being larger in supine than in both sitting and standing subjects $[3,15]$. We were not able to confirm this finding. A likely explanation of this discrepancy may be found in the difference of methods used to determine diaphragm motion. The study by Takazakura et al. [16], in fact, was conducted by using a magnetic resonance system, while another study used fluoroscopic assessment of the diaphragm [17].

In our study, the main correlates of diaphragm motion resulted to be sex and height. Although a significant correlation among height, weight, age, and diaphragm motion was found, both during $\mathrm{QB}$ and $\mathrm{DB}$ on a univariate model, only gender at $\mathrm{DB}$ and height at $\mathrm{QB}$ significantly differed on a multivariable linear model. Furthermore, we found a wide variability in diaphragmatic excursion during both $\mathrm{QB}$ and $\mathrm{DB}$, with some healthy subjects showing little or no motion but having no respiratory dysfunction or breathing discomfort. This finding represents a relevant issue: briefly, we could argue that diaphragm motion is a multifactorial ability involving many different mechanisms (such as neuromotor breathing control, muscle fatigability, myocyte metabolic reserve and contractile function, and biomechanics of breathing) and is not simply limited to mechanical and dimensional properties of the rib cage alone [18]. It is known, for example, that subjects with chronic lower back pain and sacroiliac joint pain have abnormal diaphragm movement $[19,20]$. However, it seems very likely that the relationship between height and diaphragm excursion in QB reflects the one between height and tidal volume (volume-related rib cage biomechanic properties and optimized muscle fiber's length-tension ratio), while gender affects the DP excursion due to greater maximal inspiratory and expiratory pressures produced by males (more abundant inspiratory muscle mass and physiologically higher maximal inspiratory pressures) [21].

Remarkably, we should note that our multivariable analysis failed to identify potentially relevant determinants of diaphragm variability. In fact, also including several potential anthropometric and clinical determi- 
nants such as body mass index, cigarette smoke exposure, sedentary occupational history, or physical activity, we could only ever explain a minimum amount of the total variance $(<20 \%)$. This finding is consistent with most previous studies [9]. However, we observed that, in order to establish normal diaphragm values, gender, height, but also age and weight, should be taken into account.

Although, theoretically, an accurate predictive equation of a reference value for comparison would be desirable, none of the equations we calculated were sufficiently precise to provide such an information. Further research is needed to identify those variables affecting diaphragm mobility.

The present study has some limitations. Firstly, the sample of subjects was small. Secondly, the participants were not a random sample from the adult population and had no normal distribution. However, the subjects represented most age groups (ranging from 15 to 73 years) and were balanced by sex. Thirdly, we could not test the correlation between diaphragm motion and pulmonary function indexes such as forced expiratory volume in the first second (FEV1) and forced vital capacity (FVC). Indeed, a linear correlation between lung volumes and amplitude of diaphragm motion has already been proved [9]; furthermore, Zanforlin et al. [22] have recently shown that the M-mode ultrasound forced ex- piratory profile presents similarities with the spirometric flow-volume curve. Consequently, ultrasound-derived indexes (i.e., forced diaphragmatic excursion in the first second or maximal expiratory diaphragm excursion, and the ratio of the 2) have a linear correlation with FEV1, FVC, and the FEV1/FVC ratio, both in healthy and obstructed subjects [23]. In addition, we were not able to test many variables potentially affecting the predictivity of diaphragm motion models.

In conclusion, we proved that diaphragm excursion measurements using M-mode techniques are reproducible and accurate, also when obtained in recumbent patients. Both intra- and inter-observer agreement were high enough to support the precision of these measurements and to provide a further tool for a wider application in clinical practice. After adjustments, the main predictors of diaphragm motion were sex and height, which should be taken into account to design a specifically tailored study to develop normality reference equations. Therefore, other determinants are likely to be identified to obtain highly reliable reference equations.

\section{Disclosure Statement}

All authors have no real or perceived conflict of interest to disclose.

\section{References}

1 Dubé BP, Dres M: Diaphragm dysfunction: diagnostic approaches and management Strategies. J Clin Med 2016;5:E113.

2 Sarwal A, Liu A, Cartwright MS, Dhar S, Morris PE: Sonography for assessing dynamic diaphragm dysfunction in acute respiratory distress. J Ultrasound Med 2015;34:1701-1706.

3 Leal BE, Gonçalves MA, Lisboa LG, Linné LMS, Tavares MGS, Yamaguti WP, Paulin E: Validity and reliability of fluoroscopy for digital radiography: a new way to evaluate diaphragmatic mobility. BMC Pulm Med 2017; $17: 62$.

4 Li G, Wei J, Huang H, Gaebler CP, Yuan A, Deasy JO: Automatic assessment of average diaphragm motion trajectory from 4DCT images through machine learning. Biomed Phys Eng Express 2015;1:045015.

5 Young HM, Eddy RL, Parraga G: MRI and CT lung biomarkers: towards an in vivo understanding of lung biomechanics. Clin Biomech (Bristol, Avon) 2017:S0268-0033(17) 30210-3.
6 Tanaka R: Dynamic chest radiography: flatpanel detector (FPD) based functional X-ray imaging. Radiol Phys Technol 2016;9:139153.

7 Nason LK, Walker CM, McNeeley MF, Burivong W, Fligner CL, Godwin JD: Imaging of the diaphragm: anatomy and function. Radiographics 2012;32:E51-E70.

8 Sferrazza Papa GF, Pellegrino GM, Di Marco F, Imeri G, Brochard L, Goligher E, Centanni S: A Review of the ultrasound assessment of diaphragmatic function in clinical practice. Respiration 2016;91:403-411.

9 Boussuges A, Gole Y, Blanc P: Diaphragmatic motion studied by m-mode ultrasonography: methods, reproducibility, and normal values. Chest 2009;135:391-400.

10 Testa A, Soldati G, Giannuzzi R, Berardi S, Portale G, Gentiloni Silveri N: Ultrasound Mmode assessment of diaphragmatic kinetics by anterior transverse scanning in healthy subjects. Ultrasound Med Biol 2011;37:4452.
11 Bland JM, Altman DG: Statistical methods for assessing agreement between 2 methods of clinical measurement. Lancet 1986;1:307310.

12 Hsieh KS, Lee CL, Lin CC, Huang TC, Weng $\mathrm{KP}, \mathrm{Lu} \mathrm{WH}$ : Secondary confirmation of endotracheal tube position by ultrasound image. Crit Care Med 2004;32:S374-S377.

13 Hunter SK: Sex differences in human fatigability: mechanisms and insight to physiological responses. Acta Physiol (Oxf) 2014;210: 768-789.

14 Verschakelen JA, Demedts MG: Normal thoracoabdominal motions. Influence of sex, age, posture, and breath size. Am J Respir Crit Care Med 1995;151:399-405.

15 Takazakura R, Takahashi M, Nitta N, Murata $\mathrm{K}$ : Diaphragmatic motion in the sitting and supine positions: healthy subject study using a vertically open magnetic resonance system. J Magn Reson Imaging 2004;19:605-609.
Reproducibility and Clinical Correlates of Supine Diaphragmatic Motion 
16 Takazakura R, Takahashi M, Nitta N, Sawai S, Tezuka N, Fujino S, Murata K: Assessment of diaphragmatic motion after lung resection using magnetic resonance imaging. Radiat Med 2007;25:155-163.

17 Ch'en IY, Armstrong JD 2nd: Value of fluoroscopy in patients with suspected bilateral hemidiaphragmatic paralysis. AJR Am J Roentgenol 1993;160:29-31.

18 Russo MA, Santarelli DM, O'Rourke D: The physiological effects of slow breathing in the healthy human. Breathe (Sheff) 2017;13:298309.
19 Kolar P, Sulc J, Kyncl M, Sanda J, Cakrt O, Andel R, Kumagai K, Kobesova A: Postural function of the diaphragm in persons with and without chronic low back pain. J Orthop Sports Phys Ther 2012;42:352-362.

20 Richardson CA, Snijders CJ, Hides JA, Damen L, Pas MS, Storm J: The relation between the transversus abdominis muscles, sacroiliac joint mechanics, and low back pain. Spine (Phila Pa 1976) 2002;27:399-405.

21 Clauser Pessoa IM, Franco Parreira V, Fregonezi GA, Sheel AW, Chung F, Reid WD: Reference values for maximal inspiratory pressure: a systematic review. Can Respir J 2014;21:43-50.
22 Zanforlin A, Smargiassi A, Inchingolo R, di Marco Berardino A, Valente S, Ramazzina E: Ultrasound analysis of diaphragm kinetics and the diagnosis of airway obstruction: the role of the M-mode index of obstruction. Ultrasound Med Biol 2014;40:1065-1071.

23 Zanforlin A, Smargiassi A, Inchingolo R, Valente S, Ramazzina E: Ultrasound in obstructive lung diseases: the effect of airway obstruction on diaphragm kinetics. A short pictorial essay. J Ultrasound 2014;18:379-384. 